\title{
Transcriptional Regulatory Networks in Embryonic Stem Cells
}

\author{
X. Chen, ${ }^{* \dagger}$ V.B. VegA, ${ }^{\ddagger}$ AND H.-H. NG*† \\ ${ }^{*}$ Gene Regulation Laboratory, Genome Institute of Singapore, Singapore 138672; ${ }^{\dagger}$ Department of \\ Biological Sciences, National University of Singapore, Singapore 117543; ${ }^{*}$ Computational \& \\ Mathematical Biology, Genome Institute of Singapore, Singapore 138672
}

\begin{abstract}
Embryonic stem (ES) cells are characterized by their ability to self-renew and remain pluripotent. Transcription factors have critical roles in the maintenance of ES cells through specifying an ES-cell-specific gene expression program. Deciphering the transcriptional regulatory network that describes the specific interactions of these transcription factors with the genomic template is crucial for understanding the design and key components of this network. Recent advances in genomic technologies have facilitated genome-wide disclosure of the repertoire of transcription-factor-binding sites. Transcription factor colocalization hot spots targeted by multiple transcription factors have been identified. These are sites that integrate the external signaling pathways to the transcriptional regulatory circuitry governed by Oct4, Sox2, and Nanog. In addition, these sites may serve as focal points for the assembly of nucleoprotein complexes known as enhanceosomes. Studying the properties of EScell-specific enhanceosomes in different pluripotent cells will shed light on the composition and regulation of their activity. Knowledge of the transcriptional regulatory networks in different pluripotent cells will also help to distinguish the core and peripheral parts of the networks. Collectively, these studies will facilitate the understanding of molecular mechanisms behind transcription-factor-mediated regulation of pluripotent stem cells.
\end{abstract}

Mouse ES cells are derived from the inner cell mass (ICM) of preimplantation embryos. These cells are pluripotent because they have the potential to differentiate into any cell type given the right signals. Previous work during the past decades has increased our understanding of the molecular mechanisms underlying pluripotency (Rossant 2008; Silva and Smith 2008). Several signaling and intrinsic pathways involving transcription factors have been shown to have key roles in maintaining the self-renewal and pluripotent state of ES cells (Boiani and Schöler 2005; Jaenisch and Young 2008). Leukemia inhibitory factor (LIF) has long been known to be essential for the derivation and maintenance of mouse ES cells in the presence of serum. The binding of LIF to the LIF receptor (LIFR)-gp130 heterodimer receptor on the cell membrane activates STAT3 (signal transducer and activator of transcription 3) by phosphorylation, resulting in its subsequent dimerization, nuclear translocation, and target gene activation (Niwa et al. 1998; Matsuda et al. 1999; Raz et al. 1999). However, LIF alone is not sufficient to sustain the self-renewal of ES cells in the absence of serum. Ying et al. (2003) has identified bone morphogenetic proteins (BMPs) as the growth factors that work in conjunction with LIF to promote selfrenewal in the absence of serum (Ying et al. 2003). Addition of BMP4 to chemically defined media leads to the phosphorylation of Smad1 and the activation of members of the Id (inhibitor of differentiation) gene family, which are effectors of the BMP pathway (Ying et al. 2003).

Besides these signaling pathways, which sense the presence of extrinsic growth factors in the environment, additional transcription factors are also essential for sustaining the undifferentiated state of ES cells. Oct4, Sox2, and Nanog are key components of the core regulatory network that governs ES cell pluripotency (Boyer et al.
2005; Loh et al. 2006). Other more recently identified transcriptional regulators, such as Tbx3, Esrrb, and Zfx, are also required to maintain ES cells (Ivanova et al. 2006; Loh et al. 2006; Galan-Caridad et al. 2007). Despite the critical roles of transcriptional regulators in the maintenance of mouse ES cells, in-depth knowledge of their in vivo targets is still lacking. Moreover, it is still not clear how the different transcription circuitries are connected or integrated in ES cells. Elucidation of the transcription networks that are operating in ES cells is fundamental to understand the molecular nature of pluripotency of ES cells, self-renewal of ES cells, and reprogramming of somatic cells to acquire a pluripotent state.

In this chapter, we discuss the recent advances in dissecting the transcriptional regulatory circuitries underlying self-renewal and pluripotency of mouse ES cells, with an emphasis on the emerging transcriptional regulatory network linking the key regulators.

\section{OCT4/SOX2 TRANSCRIPTION CIRCUIT}

The POU family transcription factor Oct4, which is encoded by Pou5f1 and specifically expressed in the pluripotent cells of the ICM and epiblast, acts as a gatekeeper to prevent ES cell differentiation (Nichols et al. 1998). Oct4 has been reported to regulate a set of downstream targets by forming heterodimers with Sox2 (Boiani and Schöler 2005; Chew et al. 2005; Kuroda et al. 2005; Okumura-Nakanishi et al. 2005; Rodda et al. 2005; Jaenisch and Young 2008; Silva and Smith 2008). Sox2 is a high mobility group (HMG) domain-containing transcription factor and has an expression pattern similar to that of Oct4 through mouse preimplantation development. Both Oct4 and Sox 2 null animals have primary defects in the pluripotent epiblast and both Oct4 and Sox2 
null blastocysts are incapable of giving rise to pluripotent ES cells (Nichols et al. 1998; Avilion et al. 2003).

The fine-tuning of an appropriate level of Oct4 is crucial for determining the development of distinct cell fates from ES cells. A greater than twofold increase of Oct4 causes ES cells to differentiate into primitive endoderm and mesoderm, whereas a reduction to less than $50 \%$ of normal level triggers differentiation into the trophectoderm (Niwa et al. 2000). Thus, the precise control of Oct4 levels is critical not only for the maintenance of pluripotency, but also to cell differentiation decisions.

Oct4 and Sox 2 bind to and regulate the respective regulatory regions of their own genes (Chew et al. 2005). Disruption of the circuit by short hairpin RNA (shRNA)mediated knockdown leads to the reduction of both genes' enhancer activities and endogenous expression levels and results in ES cell differentiation. Two simple network motifs are proposed to account for the Oct4-Sox2 transcription circuit: autoregulation and multicomponent loops (Boyer et al. 2005; Chew et al. 2005). The autoregulation loop is characterized by binding of the gene product to its own regulatory element, which provides a positive feedback loop and allows for self-perpetuation and enhanced stability of gene expression. The multicomponent loop is characterized by the binding of one regulator to the regulatory element of another regulator in a closed loop. This closed-circuit loop of Oct4 and Sox2 could efficiently generate a bistable system that confers on the network the capability of maintaining options between both stability and developmental switching (Boyer et al. 2005; Chew et al. 2005).

\section{NANOG/SALL4 TRANSCRIPTION CIRCUIT}

Nanog, an NK-2 class homeobox transcription factor, is another core regulator predominantly expressed in the ICM and epiblast (Chambers et al. 2003; Mitsui et al. 2003). Nanog knockout embryos fail to form epiblasts and are mostly composed of disorganized extraembryonic tissue (Chambers et al. 2003; Mitsui et al. 2003). More interestingly, overexpression of Nanog could bypass the need for LIF to maintain pluripotency in culture, suggesting a potential cross-talk between Nanog and the LIF pathway (Chambers et al. 2003).

Using affinity purification coupled to liquid chromatography-tandem mass spectrometry, Wu et al. (2006) identified Sall4, a spalt-like zinc finger protein, as an interacting partner of Nanog. The finding that Sall4 resides in the Nanog complex was also independently discovered by two other laboratories (Wang et al. 2006; Liang et al. 2008). Nanog and Sall4 were found to cooccupy common targets including the genes encoding Pou5f1, Sox2, Nanog, and Sall4 and several other important pluripotency genes (Wu et al. 2006; Zhang et al. 2006). Disruption of this circuit through shRNA-mediated knockdown causes the loss of self-renewal in ES cells, indicating that Sall4 is indispensable for the maintenance of ES cells (Zhang et al. 2006). Wu et al. (2006) also identified the autoregulatory and feedforward network motifs in the Nanog/Sall4 circuit, similar to that of the Oct4/Sox 2 circuit. Although the genome-wide cooc- cupancy of Nanog and Sall4 has not been demonstrated, it is conceivable that such cotargeting by these two factors could be extended to other target genes.

Oct4, Sox2, Nanog, and Sall4 are important components of the transcriptional regulatory network in ES cells and may function in a feedforward manner that maintains the appropriate levels of pluripotency-associated genes. Such a tightly integrated transcription circuit involving Oct4, Sox2, Nanog, and Sall4 may confer ES cells stability in resisting transient environmental noise and plasticity in a rapid response to differentiation cues. Elucidation of the downstream targets of these principle regulators is fundamental for dissecting their molecular mechanism in controlling pluripotency.

\section{CORE TRANSCRIPTIONAL REGULATORY NETWORK IN ES CELLS}

The core transcriptional regulatory circuitry consisting of Oct4, Nanog, and Sox 2 has been mapped in both human and mouse ES cells using ChIP coupled with either microarray (ChIP-on-Chip) or paired end tag sequencing (ChIP-PET) (Boyer et al. 2005; Loh et al. 2006; H.H. Ng et al., unpubl.). A striking observation that emerged from these studies is the extent of cooccupancy by all three key factors. Both studies have found that Nanog and Oct4 cooccupy considerable genomic sites. In mouse ES cells, 345 (44.5\%) Oct4 target genes are bound by Nanog (Loh et al. 2006), whereas 433 (70\%) Oct4 and Nanog cobound sites are detected in human ES cells (Boyer et al. 2005). Gene ontology analysis reveals that the cooccupied targets are enriched in both self-renewal genes and differentiation genes, suggesting that the maintenance of pluripotency could be mediated by promoting the expression of selfrenewal regulators and repressing lineage-specific developmental genes. These interesting observations suggest that these core factors converge on a set of common targets, rather than working independently, to perform their regulatory roles. The assembly of multiple factors on selective genomic fragments could assure steady gene expression of important downstream targets because transient fluctuation of any input signal would be neutralized by the other two sibling partners.

It is, however, imperative to note that protein occupancy is not necessarily linked to the regulation of the presumptive target gene. A substantial fraction of the binding sites is probably nonfunctional and may be the consequence of biological noise (Struhl 2007). It is difficult to tease apart noise from genuine regulation, but one approach is to deplete the particular gene product and attempt to infer regulation from the consequent response of the known bound genes. Loh et al. (2006) showed that a minor portion of Oct4- and Nanog-bound targets were responsive to the depletion of Oct4 and Nanog, suggesting that only a fraction of the total binding sites are functional (Loh et al. 2006). Alternatively, there could be other redundant molecules that mask the effect of depleting a single factor.

To identify the key nodes of the Oct4 and Nanog transcriptional regulatory networks, genetic manipulation is required to assess the functional roles of the candidate tar- 
get genes. Mouse ES cells depleted of Esrrb, Sall4, and Rifl, which are Oct4 and Nanog cobound targets, have been shown to lose pluripotency and undergo lineage commitment (Ivanova et al. 2006; Loh et al. 2006; Wu et al. 2006; Zhang et al. 2006). Developmental genes are preferentially up-regulated, whereas pluripotency genes are down-regulated in these knockdown cells. In another study, by coupling gene expression profiles with a ChIP data set, Matoba et al. (2006) has defined a list of 372 genes directly regulated by Oct 4 and showed by an RNA interference (RNAi) experiment that Tcl1 is required for the self-renewal of ES cells (Matoba et al. 2006). In a large-scale RNAi screening study, Ivanova et al. (2006) examined the effects of RNAi-induced knockdown of 70 genes that are preferentially up-regulated in mouse ES cells. The self-renewal capacity of ES cells was compromised after depletion of 8 of the 70 genes (Nanog, Oct4, Sox2, Tbx3, Esrrb, Tcl1, Dppa4, and Mm.343880) (Ivanova et al. 2006). More importantly, the effects could be rescued by the ectopic expression of these genes, excluding the possibility of off-target effects.

These genome-wide location analyses coupled with RNAi-mediated functional studies provide a candidate list of important genes and assist in the identification of novel regulators of self-renewal and pluripotency (Ivanova et al. 2006; Loh et al. 2006; Matoba et al. 2006). Loh et al. (2007) characterized two histone H3 lysine 9 (H3K9) demethylases of the JmjC family that are direct targets of Oct 4 and unraveled the roles of these two H3K9 demethylases in the maintenance of ES cell pluripotency (Loh et al. 2007). Jmjd2c, a histone demethylase that converts $\mathrm{H} 3 \mathrm{~K} 9$ trimethylation to dimethylation, was found to regulate the H3K9Me3 status of Nanog. Specific demethylation of H3K9Me3 by Jmjd2c at the Nanog promoter prevents the binding of transcription corepressors such as HP1 and KAP1 and sustains Nanog expression. Jmjd1a demethylates $\mathrm{H} 3 \mathrm{~K} 9 \mathrm{Me} 2$ at the Tcll promoter, a regulator of stem cell proliferation (Ivanova et al. 2006; Matoba et al. 2006). Depletion of Jmjd1a resulted in the induction of $\mathrm{H} 3 \mathrm{~K} 9 \mathrm{Me} 2$, and the altered chromatin state obstructs Oct4 from binding to the Tcll promoter. These results reveal a novel pathway in which Oct4 regulates pluripotency by activating histone modifiers that modify and maintain the expression of other Oct4 downstream target genes.

More recently, new insights have been uncovered by associating the Wnt signaling pathway with the core transcription circuitry through the terminal component Tcf3 in mouse ES cells (Pereira et al. 2006; Cole et al. 2008; Tam et al. 2008; Yi et al. 2008). Tcf3 null ES cells are unable to undergo effective differentiation in the absence of LIF (Pereira et al. 2006; Yi et al. 2008). Interestingly, Tcf3 is shown to bind to the Nanog promoter and repress its expression. Hence, elevated levels of Nanog in the absence of Tcf3 may promote the maintenance of the undifferentiated state of ES cells (Chambers et al. 2003). Genome-wide mapping of Tcf3-binding sites using ChIPon-Chip analysis revealed its global association with the Oct4, Sox2, and Nanog targets with extensive colocalization. By down-regulating Oct4, Sox2, and Nanog expression, Tcf3 is suggested to strike a balance between lineage commitment and maintenance of an undifferentiated state (Cole et al. 2008; Tam et al. 2008). Tcf3 is shown to bind to two categorically diverse sets of regulators, designated as pluripotency-associated genes and developmental genes (Cole et al. 2008; Tam et al. 2008). It is postulated that Wnt signaling impacts directly to the core regulatory network to affect the balance between pluripotency and differentiation through Tcf3 (Cole et al. 2008).

Transcription factors often recruit cofactors to modulate gene expression. The mapping of the protein interactome that defines pluripotency is pivotal for the identification of protein-protein connectivities within the transcriptional regulatory network. Two groups have explored the protein networks centered on Oct4 and Nanog using a proteomics approach (Wang et al. 2006; Liang et al. 2008). Both studies have revealed that the protein network was highly enriched for factors known to be critical for the self-renewal of ES cells and appeared to function as a module for pluripotency. Dax1, Sall4, Nac1, and Zfp281 are implicated in the maintenance of ES cell pluripotency (Wang et al. 2006). Besides transcription factors, this protein network includes cofactors found in other transcriptional repression complexes including histone deacetylase (HDAC2) and the polycomb group (YY1, Rnf2, and Rybp) (Wang et al. 2006; Liang et al. 2008). Interestingly, a unique HDAC1/2- and Mta1/2containing complex NODE (for Nanog- and Oct4-associated deacetylase) has been identified (Liang et al. 2008). This NODE complex differs from the well-known NuRD complex, with Mbd3 and Rbbp7 either absent or present at substoichiometric levels (Liang et al. 2008). Depletion of certain NODE subunits by RNAi led to increased expression of developmentally regulated genes and ES cell differentiation, highlighting the importance of these multiprotein complexes (Liang et al. 2008).

\section{EXPANDING THE TRANSCRIPTIONAL REGULATORY NETWORK IN ES CELLS}

Initial studies on the ES cell transcriptional regulatory network have revealed new insights into the structure and key nodes of this network. However, many important questions still remain unanswered. First, given the essential roles of the LIF and BMP pathways in maintaining mouse ES cell pluripotency in culture, how they are integrated into the core transcriptional network is largely unknown. Second, how the other transcription factors implicated in ES cell biology are wired into the ES cell genome is also not clear. Third, transcriptional regulation is dependent not only on transcription factors, but also on a host of coregulators. How the different coregulators are recruited to the ES cell genome to exert their roles in regulating ES-cellspecific gene expression remains unknown. Finally, the previous genome-wide mapping studies were mainly based on ChIP-on-Chip (promoter array) or ChIP-PET (paired end ditags). Due to the limited genome coverage of promoter arrays and the inadequate depth of sequence sampling by ChIP-PET, considerable binding sites of transcription factors may be missed in the previous studies.

Recent advances in ultra-high-throughput sequencing technology through massively parallel short read sequenc- 
ing (Solexa technology) have allowed the implementation of comprehensive and unbiased large-scale genome-wide mapping of transcription factors. ChIP coupled with Solexa sequencing technology (ChIP-Seq) is advantageous compared to other methods because it is simple, involves direct sequencing of small amounts of ChIP DNA, and has deeper sequencing coverage. Chen et al. (2008) have used ChIP-Seq to map the locations of 13 sequence-specific transcription factors (Nanog, Oct4, STAT3, Smad1, Sox2, Zfx, c-Myc, n-Myc, Klf4, Esrrb, Tcfcp211, E2f1, and CTCF) and two coregulators (p300 and Suz12) in ES cells. By mapping the binding sites, this study investigated the binding behavior of these factors and uncovered insights into how they are wired into the ES cell genome. More binding sites were identified by ChIPSeq as compared to ChIP-on-Chip or ChIP-PET. For example, ChIP-Seq uncovered more than 10,000 putative binding loci for Nanog, whereas ChIP-PET identified only about 3000 binding sites, with a significant fraction of the ChIP-PET identified sites inclusive in the ChIP-Seq data set (Loh et al. 2006; Chen et al. 2008). Collectively, these data sets serve as a valuable resource for understanding gene regulation in mammalian cells.

Cluster analysis of the binding sites reveals two major clusters of binding activity (Fig. 1). They are the Oct4centric and Myc-centric clusters. The Oct4-centric clusters encompass Sox2, Nanog, and Smad1. This result is consistent with the previous findings showing that they either reside in the same complex or are known to interact with one another (Boiani and Schöler 2005; Suzuki et al. 2006; Wang et al. 2006). STAT3, Esrrb, Klf4, and Tcfcp211 are also associated with Oct4-centric clusters.

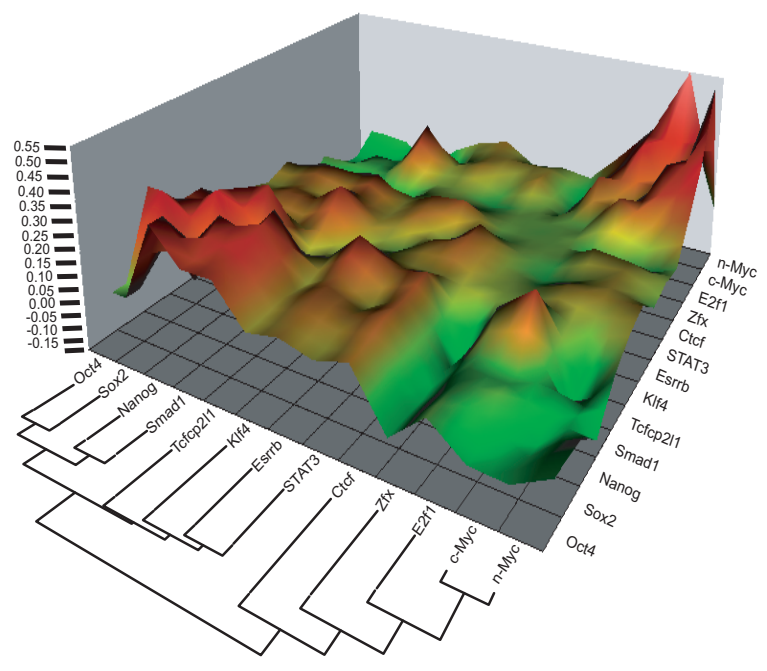

Figure 1. Cluster analysis of transcription-factor-binding sites based on 13 ChIP-Seq data sets. The transcription factor location data sets were obtained from Chen et al. (2008). The transcription factors were grouped through hierarchical clustering of the binding intensity correlation coefficient matrix calculated using the Gamma-rank correlation that measures the overall pairwise concordance and discordance over all possible pairings. The $y$ axis represents the binding intensity correlation. (Red, green) Positive and negative coefficients, respectively. The diagonal was set to zero to avoid obscuring nonidentical pairs.
On the other hand, Myc-centric clusters consist of c-Myc, $\mathrm{n}-\mathrm{Myc}, \mathrm{E} 2 \mathrm{f1}, \mathrm{Zfx}$, and CTCF. Another interesting finding is the discovery of transcription factor colocalization hot spots cooccupied by multiple transcription factors. A total of 3583 multiple transcription-factor-binding loci (MTL) were bound by four or more transcription factors. It is also of interest to note that transcription factor colocalization hot spots have also been found in other higher eukaryotes such as Drosophila (Moorman et al. 2006; Li et al. 2008). By testing 25 MTL from the Oct4-centric cluster and 8 MTL from the Myc-centric cluster for enhancer activity, Nanog-Oct4-Sox2-bound regions were found to exhibit ES-cell-specific enhancer activity, whereas Myc MTL were not active or showed very weak ES-cell-specific enhancer activity. p300 is a coactivator commonly found at enhancer regions. ChIP-Seq analysis of p300 showed that it is preferentially localized to the Oct4-centric clusters, indicative of a close relationship of p300 with the ES-cell-specific transcription circuit.

Despite the critical roles of LIF and BMP in the maintenance of mouse ES cells, the targets of the downstream effectors of these two key signaling pathways are poorly defined. It remains unresolved how the two pathways are integrated with the core transcription network. It was reported that $87.4 \%$ of Smad1-binding and $56.8 \%$ of STAT3-binding sites within MTL are associated with the Oct4-centric MTL (Chen et al. 2008). Depletion of Oct4 leads to a reduction of STAT3 and Smad1 binding on these sites, indicating that the convergence of LIF and BMP pathways with the core transcription network is mediated by an Oct4-dependent binding of STAT3 and Smad1. However, perturbation of either the LIF or BMP pathways has no effect on Oct4 binding, suggesting that Oct4 itself has a pivotal role in stabilizing this nucleoprotein complex (Chen et al. 2008).

An enhanceosome is a nucleoprotein complex composed of multiple transcription factors binding directly or indirectly to DNA (Thanos and Maniatis 1995). The enhancer of the interferon- $\beta$ gene is a prototypical enhanceosome. This 55-bp enhancer is bound by the $\mathrm{p} 50$ and $\mathrm{p} 65$ subunits of the NF- $\kappa$ B, ATF-2, IRF-3, IRF-7, and c-Jun and the architectural transcription factor HMGA (high mobility group A). The cobinding of these transcription factors is proposed to create an interface for the recruitment of coactivators such as p300. Hence, the densely occupied Oct4-centric clusters exhibit features of enhanceosomes. The proposed enhanceosomes may serve as anchor points for the relay of signaling information to the core ES cell circuitry (Fig. 2). The model of ES-cell-specific enhanceosome needs to be validated through future studies that define the DNA elements to which the transcription factors bind and ultimately confirmed by structural studies to construct the atomic model of this multiprotein/DNA complex.

The regulatory network model inferred from this study reveals both anticipated and novel links among these key transcription factors (Chen et al. 2008). Besides the regulatory feedback loops for Oct4, Sox2, Nanog, and STAT3, extensive interconnectivity was uncovered among 11 of the 13 transcription factors profiled. Moreover, several network motifs, such as the feedforward loop, biparallel motif, 


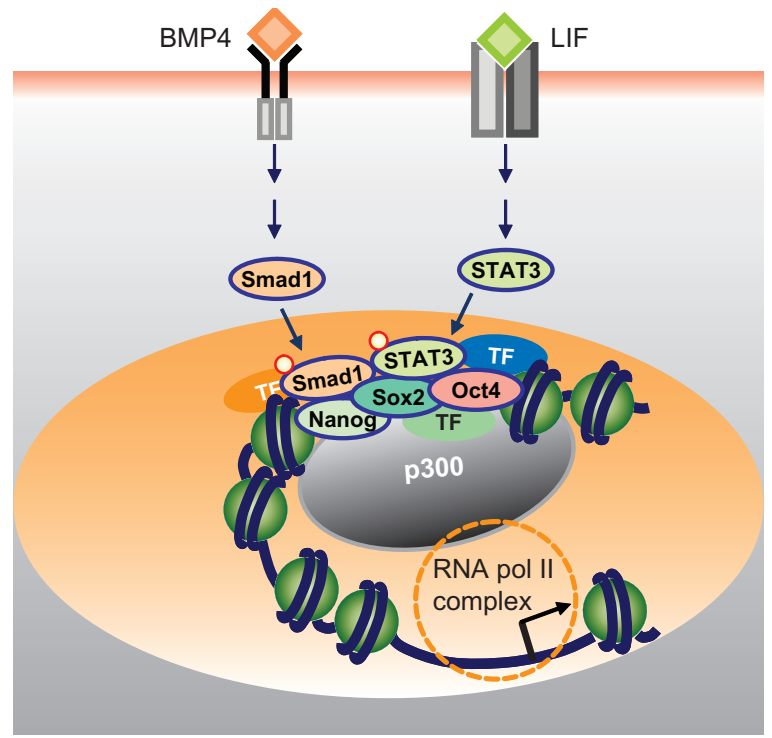

Figure 2. Integration of external signaling pathways with the core transcription network in maintaining pluripotency of mouse ES cells: A model for ES-cell-specific enhanceosome. LIF and BMP signaling pathways phosphorylate and activate nuclear translocation of STAT3 and Smad1, respectively. In the nucleus, STAT3 and Smad1 cobind specific genomic regions with Oct4, Sox2, and Nanog to form ES-cell-specific enhanceosomes. Other novel transcription factors may also be present at these sites. Coactivators such as p300 are selectively recruited to the enhanceosomes and facilitate the transcription of ES-cell-specific genes. Certain genes under the influence of these enhanceosomes may, however, be silenced by other nearby repressive cis-regulatory elements.

fully connected triad motif, and multiple input motif, were identified in the ES cell regulatory network. These building blocks of complex architecture may serve to stabilize gene expression patterns in undifferentiated ES cells.

In an independent study, Kim et al. (2008) reported the mapping of nine biotin-tagged transcription factors including Oct4, Sox2, Nanog, Klf4, c-Myc, Dax1, Rex1, Zfp281, and Nac1 using ChIP-on-Chip methodology (Kim et al. 2008). Similarly, the authors found cobinding of at least four transcription factors at 800 gene promoters. The difference in the number of multiple transcription-factor-bound loci identified could be attributed to the different factors studied and the different methodology used (ChIP-Seq versus ChIP-on-Chip). However, both groups found similar wiring patterns of the ES cell genome: the extensive colocalization of multiple transcription factors; distinct binding behavior of c-Myc from Oct4, Sox2, and Nanog; and an association of multiple binding loci to gene expression in ES cells (Chen et al. 2008; Kim et al. 2008). With these large-scale transcription factor/cofactor location maps, it is imperative to integrate these data with expression data and arrive at a regulatory code behind ES-cell-specific gene expression.

The knowledge gained in ES cells may be useful for understanding the mechanisms behind reprogramming whereby somatic cells can be reverted to pluripotent cells via coexpression of Oct4, Sox2, c-Myc, and Klf4 (Takahashi and Yamanaka 2006). Klf4 is extensively connected with Oct4, Sox2, or Nanog. It binds to the key regulatory regions of Pou $5 f 1$ and Nanog. More than $40 \%$ of Klf4 sites within the MTL clusters are also bound by Oct4, Sox 2 or Nanog. During the process of reprogramming, the wiring of the transcriptional regulatory network of a pluripotent cell needs to be reformed. It is possible that Oct4, Sox2, and Klf4 are able to jump-start the partial or entire ES cell expression program in somatic cells through targeting key nodes of the pluripotency network. Although Myc is not necessary for reprogramming, it is of interest to note that Oct4, Sox2, K1f4, and five other transcription factors bind to $\mathrm{n}-\mathrm{Myc}$, and n-Myc can replace c-Myc in reprogramming somatic cells to induced pluripotent cells (Blelloch et al. 2007). Therefore, it is conceivable that the reprogramming factors are able to activate endogenous n-Myc to form part of the transcriptional regulatory network unique to pluripotent cells. Embedded within this network is an additional level of complexity due to the presence of transcription factors exhibiting redundant functions. This is best exemplified by the finding that Klf4 alone is not important for the maintenance of ES cells (Nakatake et al. 2006). Instead, the concurrent depletion of Klf4 and two other Krüppellike factors (Klf2 and Klf5) is required for the differentiation phenotype to be manifested (Jiang et al. 2008). Hence, there is functional redundancy for the Krüppellike factors in maintaining ES cell properties.

\section{CONCLUSIONS}

Transcription factors are key for specifying gene expression programs and imparting distinct cellular phenotypes. Recent advancements in genomic technologies provide powerful platforms for a comprehensive mapping of chromatin modification profiles and binding sites of transcription factors and coregulators in ES cells (Mikkelsen et al. 2007; Chen et al. 2008). These efforts, coupled with previous knowledge of the ES cell transcriptome, will provide a framework for understanding the ES cell transcriptional regulatory networks in higher eukaryotes and facilitate a more precise association, hopefully prediction, of gene expression through the transcriptionfactor-binding pattern. In addition, these studies will greatly assist in the identification of novel self-renewal pathways, factors, and reprogramming regulators. The rapid progress made in tackling the transcriptome and genome use of ES cells will serve as a framework for further investigation to understand the biology of stem cells. With the derivation of different pluripotent cells (Rossant 2008), it will be of interest to delineate the core transcriptional regulatory network common in these cells.

\section{ACKNOWLEDGMENTS}

This work is supported by the Agency for Science, Technology, and Research (A*STAR) of Singapore. X.C. is supported by the Singapore Millennium Foundation scholarship and National University of Singapore graduate scholarship. We thank Andrew Hitchins, Jia-Hui Ng, and Wai-Leong Tam for critical comments on this manuscript. 


\section{REFERENCES}

Avilion, A.A., Nicolis, S.K., Pevny, L.H., Perez, L., Vivian, N., and Lovell-Badge, R. 2003. Multipotent cell lineages in early mouse development depend on SOX2 function. Genes Dev. 17: $126-140$.

Blelloch, R., Venere, M., Yen, J., and Ramalho-Santos, M. 2007. Generation of induced pluripotent stem cells in the absence of drug selection. Cell Stem Cell 1: 245-247.

Boiani, M. and Schöler, H.R. 2005. Regulatory networks in embryo-derived pluripotent stem cells. Nat. Rev. Mol. Cell Biol. 6: 872-884.

Boyer, L.A., Lee, T.I., Cole, M.F., Johnstone, S.E., Levine, S.S., Zucker, J.P., Guenther, M.G., Kumar, R.M., Murray, H.L., Jenner, R.G., et al. 2005. Core transcriptional regulatory circuitry in human embryonic stem cells. Cell 122: 947-956.

Chambers, I., Colby, D., Robertson, M., Nichols, J., Lee, S., Tweedie, S., and Smith, A. 2003. Functional expression cloning of Nanog, a pluripotency sustaining factor in embryonic stem cells. Cell 113: 643-655.

Chen, X., Xu, H., Yuan, P., Fang, F., Huss, M., Vega, V.B., Wong, E., Orlov, Y.L., Zhang, W., Jiang, J., et al. 2008. Integration of external signaling pathways with the core transcriptional network in embryonic stem cells. Cell 133: 1106-1117.

Chew, J.L., Loh, Y.H., Zhang, W., Chen, X., Tam, W.L., Yeap, L.S., Li, P., Ang, Y.S., Lim, B., Robson, P., and Ng, H.H. 2005. Reciprocal transcriptional regulation of Pou5f1 and Sox2 via the Oct4/Sox 2 complex in embryonic stem cells. Mol. Cell. Biol. 25: 6031-6046.

Cole, M.F., Johnstone, S.E., Newman, J.J., Kagey, M.H., and Young, R.A. 2008. Tcf3 is an integral component of the core regulatory circuitry of embryonic stem cells. Genes Dev. 22: 746-755.

Galan-Caridad, J.M., Harel, S., Arenzana, T.L., Hou, Z.E., Doetsch, F.K., Mirny, L.A., and Reizis, B. 2007. Zfx controls the self-renewal of embryonic and hematopoietic stem cells. Cell 129: 345-357.

Ivanova, N., Dobrin, R., Lu, R., Kotenko, I., Levorse, J., DeCoste, C., Schafer, X., Lun, Y., and Lemischka, I.R. 2006. Dissecting self-renewal in stem cells with RNA interference. Nature 442: 533 .

Jaenisch, R. and Young, R. 2008. Stem cells, the molecular circuitry of pluripotency and nuclear reprogramming. Cell 132: 567-582.

Jiang, J., Chan, Y.S., Loh, Y.H., Cai, J., Tong, G.Q., Lim, C.A., Robson, P., Zhong, S., and Ng, H.H. 2008. A core Klf circuitry regulates self-renewal of embryonic stem cells. Nat. Cell Biol. 10: 353-360.

Kim, J., Chu, J., Shen, X., Wang, J., and Orkin, S.H. 2008. An extended transcriptional network for pluripotency of embryonic stem cells. Cell 132: 1049-1061.

Kuroda, T., Tada, M., Kubota, H., Kimura, H., Hatano, S.Y., Suemori, H., Nakatsuji, N., and Tada, T. 2005. Octamer and Sox elements are required for transcriptional cis regulation of Nanog gene expression. Mol. Cell. Biol. 25: 2475-2485.

Li, X.Y., MacArthur, S., Bourgon, R., Nix, D., Pollard, D.A., Iyer, V.N., Hechmer, A., Simirenko, L., Stapleton, M., Luengo Hendriks, C.L., et al. 2008. Transcription factors bind thousands of active and inactive regions in the Drosophila blastoderm. PLoS Biol. 6: e27.

Liang, J., Wan, M., Zhang, Y., Gu, P., Xin, H., Jung, S.Y., Qin, J., Wong, J., Cooney, A.J., Liu, D., and Songyang, Z. 2008. Nanog and Oct4 associate with unique transcriptional repression complexes in embryonic stem cells. Nat. Cell Biol. 10: 731-739.

Loh, Y.H., Wu, Q., Chew, J.L., Vega, V.B., Zhang, W., Chen, X., Bourque, G., George, J., Leong, B., Liu, J., et al. 2006. The Oct4 and Nanog transcription network regulates pluripotency in mouse embryonic stem cells. Nat. Genet. 38: 431-440.

Loh, Y.H., Zhang, W., Chen, X., George, J., and Ng, H.H. 2007. Jmjd1a and Jmjd2c histone H3 Lys 9 demethylases regulate self-renewal in embryonic stem cells. Genes Dev. 21: $2545-2557$.

Matoba, R., Niwa, H., Masui, S., Ohtsuka, S., Carter, M.G.,
Sharov, A.A., and Ko, M.S. 2006. Dissecting Oct3/4-regulated gene networks in embryonic stem cells by expression profiling. PLoS ONE 1: e26.

Matsuda, T., Nakamura, T., Nakao, K., Arai, T., Katsuki, M., Heike, T., and Yokota, T. 1999. STAT3 activation is sufficient to maintain an undifferentiated state of mouse embryonic stem cells. EMBO J. 18: 4261-4269.

Mikkelsen, T.S., Ku, M., Jaffe, D.B., Issac, B., Lieberman, E., Giannoukos, G., Alvarez, P., Brockman, W., Kim, T.K., Koche, R.P., et al. 2007. Genome-wide maps of chromatin state in pluripotent and lineage-committed cells. Nature 448: 553-560.

Mitsui, K., Tokuzawa, Y., Itoh, H., Segawa, K., Murakami, M., Takahashi, K., Maruyama, M., Maeda, M., and Yamanaka, S. 2003. The homeoprotein Nanog is required for maintenance of pluripotency in mouse epiblast and ES cells. Cell 113: 631642 .

Moorman, C., Sun, L.V., Wang, J., de Wit, E., Talhout, W., Ward, L.D., Greil, F., Lu, X.J., White, K.P., Bussemaker, H.J., and van Steensel, B. 2006. Hotspots of transcription factor colocalization in the genome of Drosophila melanogaster. Proc. Natl. Acad. Sci. 103: 12027-12032.

Nakatake, Y., Fukui, N., Iwamatsu, Y., Masui, S., Takahashi, K., Yagi, R., Yagi, K., Miyazaki, J., Matoba, R., Ko, M.S., and Niwa, H. 2006. Klf4 cooperates with Oct3/4 and Sox2 to activate the Leftyl core promoter in embryonic stem cells. Mol. Cell. Biol. 26: 7772-7782.

Nichols, J., Zevnik, B., Anastassiadis, K., Niwa, H., KleweNebenius, D., Chambers, I., Schöler, H., and Smith, A. 1998. Formation of pluripotent stem cells in the mammalian embryo depends on the POU transcription factor Oct4. Cell 95: 379-391.

Niwa, H., Burdon, T., Chambers, I., and Smith, A. 1998. Selfrenewal of pluripotent embryonic stem cells is mediated via activation of STAT3. Genes Dev. 12: 2048-2060.

Niwa, H., Miyazaki, J., and Smith, A.G. 2000. Quantitative expression of Oct-3/4 defines differentiation, dedifferentiation or self-renewal of ES cells. Nat. Genet. 24: 372-376.

Okumura-Nakanishi, S., Saito, M., Niwa, H., and Ishikawa, F. 2005. Oct-3/4 and Sox 2 regulate Oct-3/4 gene in embryonic stem cells. J. Biol. Chem. 280: 5307-5317.

Pereira, L., Yi, F., and Merrill, B.J. 2006. Repression of Nanog gene transcription by Tcf3 limits embryonic stem cell selfrenewal. Mol. Cell. Biol. 26: 7479-7491.

Raz, R., Lee, C.K., Cannizzaro, L.A., d'Eustachio, P., and Levy, D.E. 1999. Essential role of STAT3 for embryonic stem cell pluripotency. Proc. Natl. Acad. Sci. 96: 2846-2851.

Rodda, D.J., Chew, J.L., Lim, L.H., Loh, Y.H., Wang, B., Ng, H.H., and Robson, P. 2005. Transcriptional regulation of Nanog by OCT4 and SOX2. J. Biol. Chem. 280: 24731-24737.

Rossant, J. 2008. Stem cells and early lineage development. Cell 132: $527-531$

Silva, J. and Smith, A. 2008. Capturing pluripotency. Cell 132: 532-536.

Struhl, K. 2007. Transcriptional noise and the fidelity of initiation by RNA polymerase II. Nat. Struct. Mol. Biol. 14: 103-105.

Suzuki, A., Raya, A., Kawakami, Y., Morita, M., Matsui, T., Nakashima, K., Gage, F.H., Rodríguez-Esteban, C., and Izpisúa Belmonte, J.C. 2006. Nanog binds to Smad1 and blocks bone morphogenetic protein-induced differentiation of embryonic stem cells. Proc. Natl. Acad. Sci. 103: 10294-10299.

Takahashi, K. and Yamanaka, S. 2006. Induction of pluripotent stem cells from mouse embryonic and adult fibroblast cultures by defined factors. Cell 126: 663-676.

Tam, W.L., Lim, C.Y., Han, J., Zhang, J., Ang, Y.S., Ng, H.H., Yang, H., and Lim, B. 2008. Tcf3 regulates embryonic stem cell pluripotency and self-renewal by the transcriptional control of multiple lineage pathways. Stem Cells 26: 2019-2031.

Thanos, D. and Maniatis, T. 1995. Virus induction of human IFN $\beta$ gene expression requires the assembly of an enhanceosome. Cell 83: 1091-1100.

Wang, J., Rao, S., Chu, J., Shen, X., Levasseur, D.N., Theunissen, T.W., and Orkin, S.H. 2006. A protein interaction network for pluripotency of embryonic stem cells. Nature 444: 364-368. 


\section{REGULATORY NETWORKS IN EMBRYONIC STEM CELLS}

Wu, Q., Chen, X., Zhang, J., Loh, Y.H., Low, T.Y., Zhang, W., Zhang, W., Sze, S.K., Lim, B., and Ng, H.H. 2006. Sall4 interacts with Nanog and co-occupies Nanog genomic sites in embryonic stem cells. J. Biol. Chem. 281: 24090-24094.

Yi, F., Pereira, L., and Merrill, B.J. 2008. Tcf3 functions as a steady state limiter of transcriptional programs of mouse embryonic stem cell self renewal. Stem Cells 26: 1951-1960. Ying, Q.L., Nichols, J., Chambers, I., and Smith, A. 2003. BMP induction of Id proteins suppresses differentiation and sustains embryonic stem cell self-renewal in collaboration with STAT3. Cell 115: 281-292.

Zhang, J., Tam, W.L., Tong, G.Q., Wu, Q., Chan, H.Y., Soh, B.S., Lou, Y., Yang, J., Ma, Y., Chai, L., et al. 2006. Sall4 modulates embryonic stem cell pluripotency and early embryonic development by the transcriptional regulation of Pou $5 f 1$. Nat. Cell Biol. 8: 1114-1123. 


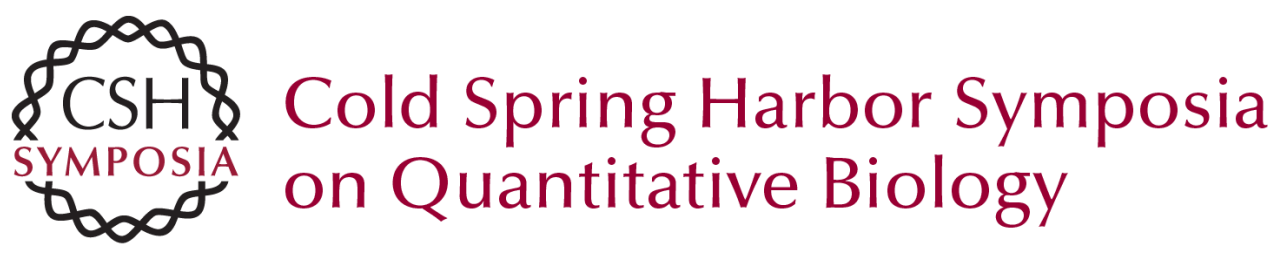

\section{Transcriptional Regulatory Networks in Embryonic Stem Cells}

X. Chen, V.B. Vega and H.-H. Ng

Cold Spring Harb Symp Quant Biol 2008 73: 203-209 originally published online November 6, 2008 Access the most recent version at doi:10.1101/sqb.2008.73.026

References This article cites 43 articles, 15 of which can be accessed free at: http://symposium.cshlp.org/content/73/203.full.html\#ref-list-1

\section{License}

Email Alerting Receive free email alerts when new articles cite this article - sign up in the Service box at the top right corner of the article or click here. 